\title{
Feasibility of MRI in Patients with Non-Pacemaker/Defibrillator Metallic Devices and Abandoned Leads
}

\author{
Prabhakaran P. Gopalakrishnan $^{1}$ (D), Loretta Gevenosky², Robert W. W. Biederman ${ }^{2}$ \\ ${ }^{1}$ Division of Cardiology, Aultman Deuble Heart and Vascular Hospital, Canton, OH, USA; ${ }^{2}$ Cardiovascular MRI \\ Center, Allegheny Health Network Cardiovascular Institute, Pittsburgh, PA, USA
}

Correspondence to: Robert W. W. Biederman, Robert.Biederman@ahn.org

Keywords: MRI Safety, Pacemaker, Abandoned Leads, Implantable Loop Recorder, Extra-Cardiac Devices

Received: December 2, $2020 \quad$ Accepted: March 6, $2021 \quad$ Published: March 9, 2021

Copyright $\odot 2021$ by author(s) and Scientific Research Publishing Inc.

This work is licensed under the Creative Commons Attribution International License (CC BY 4.0).

http://creativecommons.org/licenses/by/4.0/

\section{(c) (i) Open Access}

\section{ABSTRACT}

Objective: To evaluate feasibility of MRI in patients with non-pacemaker (PM)/Implantable cardioverter defibrillator (ICD) metallic devices and abandoned leads. Background: Relative safety of MRI performed using specified protocol has been established in MR non-conditional PM/ICDs. With limited safety data, many non-PM/ICD metallic devices and abandoned leads continue to be a contraindication for MRI. Methods: We retrospectively analyzed consecutive patients with extra-cardiac devices, non-programmable cardiac devices, and abandoned leads, who underwent MRI (GE 1.5 Tesla, WI) at a single tertiary care center over a span of 13 years. Scan protocol was designed to maintain specific absorption rate $(\mathrm{SAR})<4.0 \mathrm{~W} / \mathrm{kg}$ and scan time $<60$ minutes. Results: The cohort comprised $127 \mathrm{MRI}$ exams representing 94 patients, with 13 patients having two or more scans. The devices consisted of: 23 vagal nerve stimulators (VNS), 22 implantable loop recorders, 16 spinal stimulators, 5 peripheral nerve stimulators, 3 bladder stimulators, 2 deep brain stimulators, 1 gastric stimulator, 1 bone stimulator, 1 WATCHMAN device, 22 abandoned PM/1CD leads and 1 VNS lead. There was no immediate (peri-MRI exam) morbidity or mortality. Patients did not report any discomfort, palpitations, heating, or sensation of device migration during the exam. Local follow-up data was available in $65 \%$ (100\% for thoracic imaging) with a mean of $190 \pm 475$ days (median 13 days). No device malfunction was reported during follow-up. Conclusions: With appropriate precautions, MRI is feasible in patients with extracardiac devices, nonprogrammable cardiac devices, and abandoned leads. 


\section{INTRODUCTION}

Magnetic resonance imaging (MRI) has come a long way since the first human MRI scan in 1977 by Damadian et al. [1]. Despite significant technological advances in MR imaging, safety concerns have limited its use in patients with metallic devices, in vast majority of institutions. The 2008 Joint Commission Sentinel Event Issue 38 regarding MRI safety was sweeping and states "In general, do not bring any device or equipment into the MRI environment unless it is proven to be MR Safe or MR Conditional" [2]. This alert has since been retired and replaced by a more nuanced guidance released in 2013 from the American College of Radiology (ACR) [3], which per se is the second update for the initial MRI safety guidance released by ACR in 2002. The growing utility and ubiquity of cardiac and non-cardiac devices has necessitated studying MRI safety in patients with these devices. Indeed, MRI safety in patients with metallic objects has been studied since early years of clinical MR imaging [4-6]. Until recently, regulatory agencies considered programmable cardiac implantable electronic devices (CIEDs) such as pacemakers and implantable cardioverter defibrillators (PM/ICD) as a contraindication for MRI [7]. In 2011, the Food and Drug Administration approved the first MR conditional pacemaker [8]. Recent work has suggested that with an appropriate protocol, MRI in patients with legacy CIEDs (MR non-conditional PM/ICDs) may be safer than previously believed. In 2011, Nazarian et al. reported safety of MRI with appropriate precautions in a series of 438 patients with programmable cardiac devices (54\% PMs and 46\% ICDs) [9]. The landmark MagnaSafe Registry, a multicenter prospective cohort study, evaluated 1500 patients with programmable CIEDs implanted after 2001 (1000 PMs, 500 ICDs) [10] with no morbidity or mortality. Patients underwent non-thoracic MRI at $1.5 \mathrm{Tesla}(\mathrm{T})$ after appropriate screening and reprogramming based on a pre-specified protocol [11]. No mortality, lead failure, loss of capture or ventricular arrhythmia was reported during the scan [10]. Only an isolated ICD generator which was not reprogrammed per specification before the scan had to be replaced. This study overwhelmingly demonstrated safety of $1.5 \mathrm{~T}$ in non-conditional PM/ICDs, provided the devices were reprogrammed according to pre-specified protocol. More recently, a single center prospective study of $1.5 \mathrm{~T}$ MRI in 1509 patients with legacy MR non-conditional PM/ICDs (2103 MRI exams) also showed safety of MRI in these patients [12]. While safety issues continue to be addressed, the additive diagnostic value of MRI in patients with PM/ICDs has been addressed as well. We recently published a single center study of 136 patients with CIEDs undergoing MRI demonstrating, the additive value of MRI to diagnosis and management in $97 \%$ of the patients without any safety issues [13]. In 30\% of the patients, principle diagnosis and subsequent management changed after MRI, demonstrating that beyond safety, a bona fide protocol can catalyze appropriate management supporting more widespread adoption of this approach.

While the general field of MR imaging in patients with PM/ICD devices has gradually matured over the last two decades to a semblance of acceptability, when performed at centers of expertise and onlywhen the risk/benefit ratio permits, there remains widespread reticence for universal acceptability. Until recently, PM/ICD's remained a relative contraindication unless the patients were to have a conditional device. Thus, it naturally follows that safety of other metallic devices and abandoned PM/ICD leads which are not amenable for manipulation to MRI safe or safer modes, is far from clear. Indeed, due to lack of programmability some of these devices are historically felt to be at the highest risk for MRI scanning. Consequently, this represents nearly an absolute contraindication in most guidelines and practices. With greater than 125,000 sacral nerve modulators, 100,000 deep brain stimulators and 75,000 vagal nerve stimulators reportedly implanted [14], many of these patients will have need for MRI at some stage in their lifetime. In addition to this large population of extra-cardiac devices, there is a sizeable population with abandoned pacemaker/ICD leads, implantable loop recorders and other non-programmable cardiac devices. Data on safety evaluation for MRI in patients with these non-PM/ICD metallic devices and leads is limited to non-clinical testing, retrospective studies, or anecdotes. 2013 ACR guidance on MRI safety states, patients with "long conductive leads, pacemakers, ICDs, neurostimulators, and cochlear implants, should be considered at risk for MR studies if the body coil is to be used for RF transmission over the region of the electrically conductive lead, even if only part of the lead pathway is within the volume to undergo RF irradia- 
tion" [3]. The 2017 Heart Rhythm Society expert consensus statement on MRI and radiation exposure in patients with CIEDs, states, "in presence of abandoned leads even MRI conditional devices have to be considered as MRI non-conditional” [15]. In January 2018, based on MagnaSafe and other studies, Medicare has extended coverage for MRI to include patients with MR non-conditional PM/ICDs who are not pacemaker dependent but still explicitly excludes patients with "fractured, epicardial, or abandoned leads" [16] specifically stating the data was too "sparse" to render an opinion while intimating the need for further safety data. Herein, we report a single center experience on the feasibility of MRI in patients with non-PM/ICD metallic devices and leads.

\section{HYPOTHESIS}

We hypothesize that using an established protocol analogous to that employed in PM/ICD's, non-programmable devices, non-cardiac devices, and abandoned leads can be imaged in a $1.5 \mathrm{~T}$ MRI with acceptable procedural and post-procedural safety.

\section{METHODS}

We performed a retrospective analysis of consecutive patients with non-programmable cardiac devices (implantable loop recorders, left atrial appendage occlusion device (LAAO), etc.), extra-cardiac devices (vagal nerve stimulators, deep brain stimulators, gastric stimulators, bladder stimulators, etc.) and abandoned PM/ICD leads, who underwent MRI (GE 1.5 T, WI) at a single tertiary care center over a span of 13 years (Feb 2006-Mar 2019). All patients referred for MRI had a valid clinical indication, with $>95 \%$ of the referred patients having undergone the requested imaging study. Roguin et al. reported that pacemakers and ICDs manufactured after 2000 were probably safe in MRI environment due to device improvements [17]. Based on this, the MagnaSafe study used an empiric implantation date cutoff of 2002 for study inclusion. In abundance of caution, in our study, only patients with devices and leads that were implanted after 2004 were imaged. All patients gave written informed consent in a separate clinic witnessed by a family member after a detailed discussion with the imaging cardiologist performing the study, regarding known risk and benefits. Patients were informed about the limited clinical experience with MRI in non-PM/ICD devices and abandoned leads as an integral part of the consent process. For the purpose of reducing induced currents (from gradient magnetic fields and RF potentials) and heating (from RF potentials), the MRI scan protocol was designed to maintain specific absorbance rate (SAR) less than $4.0 \mathrm{~W} / \mathrm{kg}$ (with preferred SAR of less than $2.0 \mathrm{~W} / \mathrm{kg}$ ) and limit study duration to less than 60 minutes, similar to the protocol successfully used for intact PM/ICDs in MagnaSafe study and our recent work [13]. Patients with projectiles (bullets, shrapnel etc.) were excluded. Vital signs and EKG were monitored. The patients were under constant audio, visual and electronic monitoring during the study for any evidence of distress or change in vital signs. A cardiologist with training in advanced cardiac life support was in attendance during the study. No specific device interrogation or programming was performed prior to or after the MRI scan, other than switching to and from MRI mode in devices with that capability.

\section{RESULTS}

The cohort comprised of 127 MRI exams representing 94 patients, spanning 13 years from Feb 2006 through Mar 2019. Thirteen patients had repeat ( 2 or more) scans, including one patient with abandoned leads who had 9 MRI scans. Average study duration was $35 \pm 9$ min. The devices consisted of: 23 vagal nerve stimulators (VNS), 22 implantable loop recorders, 16 spinal stimulators, 5 peripheral nerve stimulators, 3 bladder stimulators, 2 deep brain stimulators, 1 gastric stimulator, 1 bone stimulator, 1 WATCHMAN device, 22 abandoned PM/lCD leads and 1 VNS lead. Three patients had multiple non-PM/ICD metallic devices (two patients with ILR and abandoned leads and another patient with ILR and VNS) (Table 1). This represented 58 neuro/neurosurgical, 28 cardiovascular (including 4 post-cardiac transplant patients) and 8 musculoskeletal studies. Some portion of the non-PM/ICD metallic device or leads was within the region imaged in at least 38 out of 94 patients and 51 out of 127 scans (Figure 1). None of the patients 
Non-pacemaker/defibrillator metallic devices and abandoned leads in MRI environment
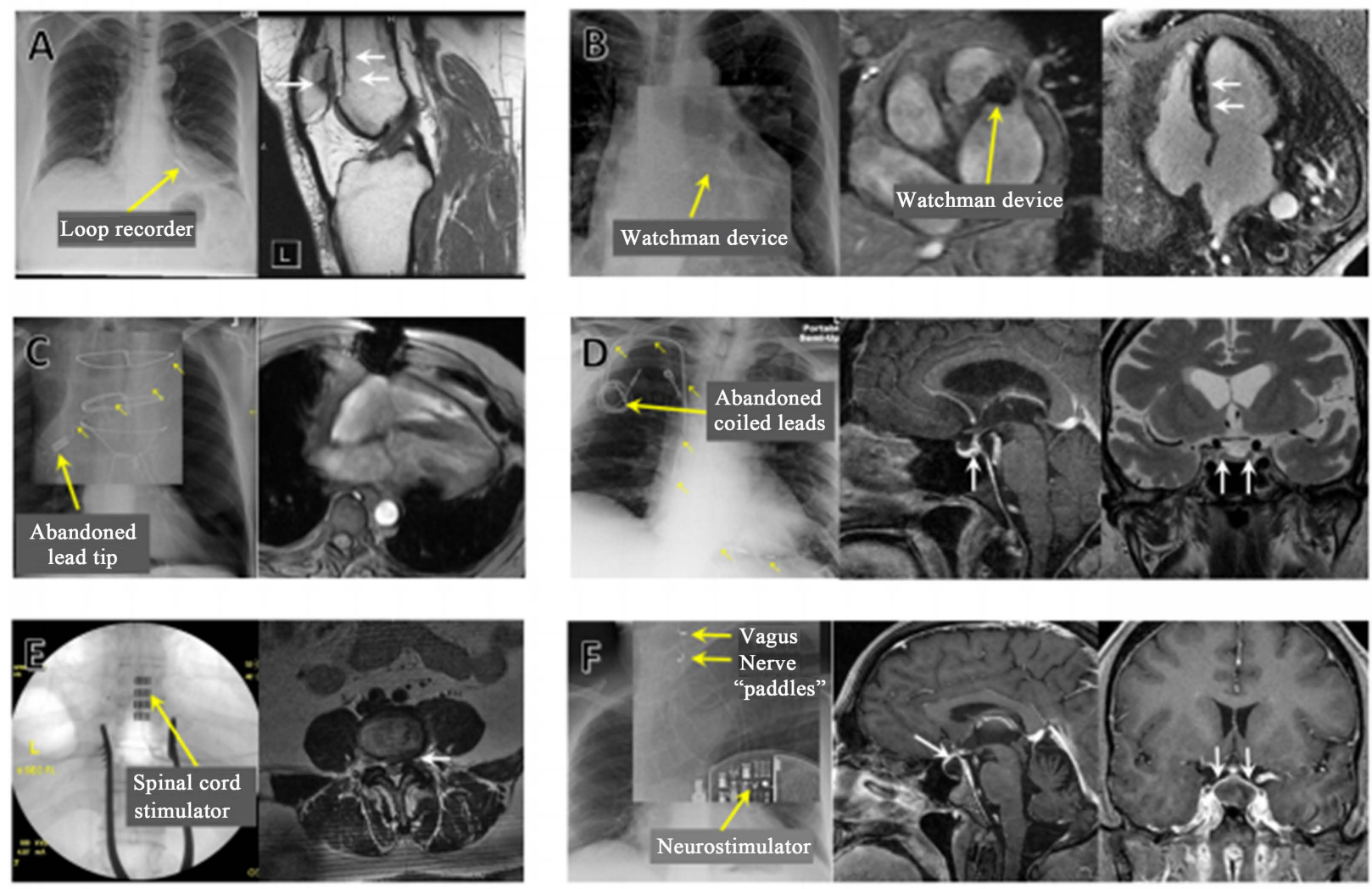

Figure 1. Non-pacemaker/defibrillator metallic devices and abandoned leads in MRI environment. Yellow arrows: Device/lead; White arrows: Pathology identified. A. MRI of left knee in a patient with implantable loop recorder, showing mild tricompartmental chondrosis. B. Cardiac MRI in a nonischemic cardiomyopathy patient with a Watchman device, showing delayed hyperenhancement in inferoseptal segment. C. Cardiac MRI in a heart transplant patient with retained leads, presenting with chronic rejection. D. MRI brain in a patient with coiled abandoned leads, showing a hypoenhancing lesion in pituitary gland and pituitary compression. E. MRI lumbar spine in a patient with spinal cord stimulator, showing disc herniation with spinal canal and foraminal stenosis. F. MRI Brain in a patient with vagal nerve stimulator, showing cystic lesion in sella turcica with suprasellar extension.

reported any discomfort, palpitations, heating, or sensation of device migration at the time of study or in the 30-minute post-scan period. There was no immediate (peri-MRI exam) morbidity or mortality. Local follow-up data was available in $65 \%$ (100\% for thoracic imaging) with a mean of $190 \pm 475$ days (median 13 days; range: 1 day - 10.9 years). None of these patients $(0 \%)$ had device malfunction (indicated by device malfunction or alerts) at the time of short- or long-term follow-up. Importantly, with careful attention to patient positioning and scan sequences, no safety or device issues were encountered in any patient, including the ten patients who underwent repeat scans.

\section{DISCUSSION}

The hazards of MRI in patients with metallic devices are considered, in part, to be related to the main static magnetic field $B_{0}$ (torque), gradient magnetic field (induced currents) or radiofrequency pulse (induced currents, heating from antenna effect) [18]. PM/ICDs, the most well studied device category in MR 
Table 1. Non-PM/ICD devices and abandoned leads.

\begin{tabular}{cccc}
\hline Device & Number of patients & Number of scans & Mean follow-up (days) \\
\hline Vagal nerve stimulator (VNS) & $23^{*}$ & $34^{*}$ & $196^{*}$ \\
Implantable loop recorder (ILR) & $22^{*}$ & $26^{*}$ & $84^{*}$ \\
Abandoned PM/ICD leads & $22^{*}$ & $38^{*}$ & $426^{*}$ \\
Spinal cord stimulator & 16 & 16 & 46 \\
Peripheral nerve stimulator & 5 & 5 & 65 \\
Bladder stimulator & 3 & 5 & 324 \\
Deep brain stimulator & 2 & 2 & 240 \\
Gastric stimulator & 1 & 1 & 0 \\
Bone stimulator & 1 & 1 & 0 \\
Abandoned VNS wires & 1 & 1 & 196 \\
Watchman device & 1 & 1 & 16 \\
All Devices & $94^{*}$ & $127^{\star}$ & $190^{\star}$ \\
\hline
\end{tabular}

${ }^{\star}$ One patient had both VNS and ILR and two patients had ILR and abandoned leads.

environment, have undergone design changes to improve their MRI safety. These changes include reducing the ferromagnetic content, changes in lead design to minimize heating and facilitate heat dissipation, hermetically sealed titanium, or stainless-steel cases, etc. [18]. Recent studies have shown with appropriate scan protocol and program settings, MRI can be safely (but not with impunity) performed in older MR non-conditional PM/ICDs as well [10]. This still leaves a large group of patients who have non-programmable cardiac devices, abandoned PM/ICD leads and a wide spectrum of extra-cardiac devices.

Non-programmable cardiac devices such as ILRs have not been adequately studied in clinical settings. Even less attention has been paid to abandoned pacemaker or ICD leads and occlusion devices. Most current generation ILRs are generally considered MR conditional (based on non-clinical testing), although device memory/ECG recording will be inaccurate during scanning [18]. Artifacts during MRI may mimic arrhythmia $[19,20]$. Occlusion devices such as Amplatzer plug and WATCHMAN device are considered MR conditional based on ex vivo testing [21]. Abandoned leads are disconnected from the pulse generator and left behind due to fracture, insulation breaks, dislodgment or other failure [22]. Lead extraction is fraught with high risk that they are often not removed even at the time of open-heart surgery. Studies show $39 \%$ to $48 \%$ of post heart transplant patients had abandoned leads even after transplant surgery from devices (ICDs and cardiac resynchronization therapy devices) placed in their native heart [23, 24]. The wavelength in tissue of RF for $1.5 \mathrm{~T}$ MRI is $52 \mathrm{~cm}$ which falls in clinically relevant lead length range of 40 $60 \mathrm{~cm}$, increasing chance of antenna effect related heating [22]. Abandoned leads have been shown to exhibit increased lead tip heating compared to pacemaker-attached leads [22]. In addition, capped leads have been shown to be more prone for heating than uncapped leads [25]. Even if the lead is MR compatible by design, abandoned leads are considered MR non-conditional and MRI is not recommended [15, 25]. The pacing capture threshold, typically used to assess myocardial damage due to MRI exposure, cannot be measured for abandoned leads [22]. While myocardial damage leading to under sensing or failure to capture is not a problem with abandoned leads, myocardial damage and scarring may have detrimental effects on cardiac function as well as have a pro arrhythmic effect.

The extra-cardiac devices currently being used clinically include spinal cord stimulators, deep brain stimulators, sacral nerve modulators, vagal nerve stimulators, gastric stimulators, phrenic nerve stimula- 
tors, bone stimulators and carotid sinus stimulators [14]. Prevalence of these devices in patients has been expanding. These extra-cardiac devices have a wide range of properties, anatomic location, and programmability. Some of them are programmable and some are not. In some devices, the programming could be performed by the patient and in some by the physician only and typically only to "on" or "off" modes. Data for MRI safety in these devices is limited to non-clinical testing and retrospective studies. Neurological damage has been reported in a patient with deep brain stimulator undergoing brain MRI [26].

The landmark MagnaSafe study on MRI safety in MR non-conditional PM/ICDs, which one of the authors participated in, showed MRI could be performed safely in these patients using appropriate MRI protocols and programming the devices to specified modes. Importantly, the MagnaSafe study specifically excluded patients with abandoned or inactive leads that could not be interrogated, non-PM/ICD devices or devices with non-thoracic location [10]. Published safety evaluation for MRI in patients with non-PM/ICD metallic devices is very limited.

A very small study of 2 patients with ILRs showed no safety issue with MRI in either patient [27]. Another small retrospective cohort study of 19 patients with leads who underwent MRI ( 35 scans) found no safety concerns after a 7-day follow-up [28]. Incredibly, these patients had their generators removed prior to MRI and re-implanted after the MRI scan. At the time of this study the generator was perceived to be more of a risk in the MRI environment than the leads. Based on the safety demonstrated during MagnaSafe study as well our single center experience with CIEDs, we believe the risk associated with PM/ICD removal far exceeds the risk of MRI performance. At minimum, our experience and MagnaSafe data should suggest that MRI with the device in situ may be a far more conservative strategy than device explantation for sole purpose of performing MRI and such should be considered in the future as a preferred strategy. Strach et al. reported a series of 114 patients with CIEDs including an unknown number of patients with abandoned leads with no safety issues when imaged with $0.5 \mathrm{~T}$ MRI, but data on safety in the more commonly used field strength of $1.5 \mathrm{~T}$ is limited [29]. Safety of permanent epicardial surgical leads in the MRI environment is not well understood. A study of 200 patients with postoperative metallic material (including 51 patients with abandoned temporary epicardial pacing wires) who underwent 1.0 or 1.5 T MRI after cardiac surgery did not show any symptoms suggestive of arrhythmia or cardiac dysfunction. Only 81 of the 200 patients had ECG monitoring during the scan [30]. No intermediate or long-term follow-up was performed. A MR safety survey of directors of 105 neuroradiology fellowship programs in US and Canada did not find any safety issues for MRI in patients with abandoned epicardial pacer wires [31]. However, other investigators recommend caution [32]. Our own experience is that in actual practice, most centers express considerable reticence towards imaging any metallic device.

A Dutch study of 73 patients with VNS who underwent MRI (101 scans) demonstrated no complications except for one instance of a lead break but it was unclear if MR scanning was responsible for the lead break [33]. Blood oxygenation level-dependent functional MR imaging (BOLD fMRI) synchronized to pulses from a VNS has been shown to be feasible [34]. A study of 21 patients with LAAO devices (Amplatzer, Amulet and WATCHMAN) who underwent MRI brain within 48 hours after procedure did not report any safety issues [35]. A series of 7 patients with LAAO devices who underwent cardiac MRI with contrast to evaluate placement did not report any safety issues [36].

While small studies have reported safety of MRI in individual devices, our study comprised an array of non-PM/ICD metallic devices and abandoned leads. There were no safety issues during the scan or in the immediate post scan monitoring period. In more than a third of the scans, the entire device or abandoned lead or a portion of it was within the imaging field. None of the patients who had follow-up (79\%), had any safety issue at the time of follow-up. Padmanabhan et al. [37] reported a favorable risk-benefit profile for MRI in patients with abandoned leads. Based on data from our lab, Samar et al. [13] previously demonstrated additive value of MRI in management of patients with programmable cardiac devices. We believe our findings add to growing evidence demonstrating feasibility of MRI in patients with a wide spectrum of devices - cardiac and extra-cardiac, programmable and non-programmable, provided appropriate MRI protocol is used. This approach will avoid compromising patient care by having to resort to investigations with lesser diagnostic value or more invasive strategies with higher inherent risk, e.g., re- 
moval of devices or leads to facilitate MRI, invasive diagnostic approaches such as biopsy in lieu of MRI. While physicians are bound by Hippocratic Oath of "primum non nocere" in being cautious about MRI in patients with metallic devices, there is counter argument against depriving patients of this important diagnostic tool, shown to have impact on survival in certain conditions [38].

In 2011, the Centers for Medicare and Medicaid Services (CMS) granted a change in National Coverage Determination (NCD) that allowed reimbursement for MRI in patients with PM/ICD if they are enrolled in a prospective registry designed to determine the risk associated with MRI [39]. In view of expanding data on safety of MRI in patients with devices, there was clamor for re-examining of coverage issues [40]. In January 2018, CMS extended coverage for MRI to include patients with MR non-conditional PM/ICDs if following criteria are met: $\leq 1.5$ Tesla; $\geq 6$ weeks after device placement; not pacemaker dependent; no fractured, epicardial, or abandoned leads; programming device to appropriate MRI scanning mode [16]. We strongly believe our experience will expand current understanding in this field, as our study includes the patients specifically excluded by CMS in the recent NCD due to limited available data: abandoned leads, devices lacking programmability to MRI-safe mode.

While MRI safety in older MRI non-conditional cardiac devices as well as non-cardiac devices is being established, device improvements to make these devices MR conditional have been developed. It is noteworthy that improvements in device and lead design to make them MRI conditional have their pitfalls as well. Several studies have raised concerns about increased risk of lead dislodgement and cardiac perforation with newer MR conditional pacing leads [41, 42]. This suggests replacing MR non-conditional devices with newer MR conditional devices for sole purpose of MRI is not only necessary but may be fraught with additional risks beyond the inherent peri-procedural complications.

While our study serves to expand the knowledge of MRI safety in patients with non-PM/ICD metallic devices, it has several limitations. The devices in our study were not interrogated before or after the MRI exam. While the great majority of the patients (75\%) had follow-up, local follow-up data was not available for patients referred from outside centers. While no cardiovascular, neurological or other adverse effects were noted during the scan, immediately after the scan or during follow-up, lack of data regarding the performance of the devices in patients without long term follow-up is a limitation, notwithstanding the notion that peri-procedural and short-term follow-up likely encapsulates the great majority of risk. While our study represents the largest real world study representing a wide spectrum of non-cardiac devices, non-programmable cardiac devices and abandoned leads to our knowledge, the small sample size for some of the individual devices ( 2 bladder stimulator, 2 deep brain stimulator, 1 bone stimulator, WATCHMAN device etc.) may limit applicability of our data for those devices. This is a single center retrospective study at a Cardiovascular MRI lab with long experience in imaging patients with implantable devices. Accordingly, caution is needed while extrapolating our findings to centers with less experience in imaging these patients. Replicating this data in a multicenter prospective study would further strengthen the evidence for MRI safety in non-PM/ICD metallic devices and abandoned leads should clinical equipoise remain.

\section{CONCLUSION}

In this early experience, we show that MRI is feasible in patients with implantable loop recorders, an assortment of stimulators and abandoned $\mathrm{PM} / \mathrm{lCD}$ leads, when combined with scan protocols used for patients with intact MR non-conditional PM/ICDs. Unlike most studies which focused on a particular device, our study represents the largest to date real world experience demonstrating feasibility and early favorable safety of MR imaging in patients with a wide array of non-PM/ICD metallic devices encountered in clinical setting. With the previously demonstrated notion of "added value" for MRI in PM/ICD's, the feasibility of MRI with appropriate precautions that we have demonstrated in patients with non-PM/ICD metallic devices and abandoned leads, should spur further research in this field.

\section{AUTHOR CONTRIBUTIONS}

PG—Data collection and analysis, drafting article; LG-Data collection and critical revision of article; 
$\mathrm{RB}-$ Concept/design, data analysis, critical revision of article and approval of article.

\section{CONFLICTS OF INTEREST}

The authors declare no conflicts of interest regarding the publication of this paper.

\section{REFERENCES}

1. Damadian, R., Goldsmith, M. and Minkoff, L. (1977) NMR in Cancer: XVI. FONAR Image of the Live Human Body. Physiological Chemistry and Physics, 9, 97-100, 108.

2. (2008) Sentinel Event Alert, Issue 38: Preventing Accidents and Injuries in the MRI Suite.

3. Expert Panel on MRS, Kanal, E., Barkovich, A.J., Bell, C., Borgstede, J.P., Bradley, W.G., et al. (2013) ACR Guidance Document on MR Safe Practices: 2013. Journal of Magnetic Resonance Imaging, 37, 501-530. https://doi.org/10.1002/jmri.24011

4. Davis, P.L., Crooks, L., Arakawa, M., McRee, R., Kaufman, L. and Margulis, A.R. (1981) Potential Hazards in NMR Imaging: Heating Effects of Changing Magnetic Fields and RF Fields on Small Metallic Implants. American Journal of Roentgenology, 137, 857-860. https://doi.org/10.2214/ajr.137.4.857

5. Zheutlin, J.D., Thompson, J.T. and Shofner, R.S. (1987) The Safety of Magnetic Resonance Imaging with Intraorbital Metallic Objects after Retinal Reattachment or Trauma. American Journal of Ophthalmology, 103, 831. https://doi.org/10.1016/S0002-9394(14)74404-6

6. Shellock, F.G. (2002) Magnetic Resonance Safety Update 2002: Implants and Devices. Journal of Magnetic Resonance Imaging, 16, 485-496. https://doi.org/10.1002/jmri.10196

7. Faris, O.P. and Shein, M.J. (2005) Government Viewpoint: U.S. Food \& Drug Administration: Pacemakers, ICDs and MRI. Pacing and Clinical Electrophysiology, 28, 268-269. https://doi.org/10.1111/j.1540-8159.2005.50035.x

8. (2011) FDA Approves First Pacemaker System Safe for Magnetic Resonance Imaging. Traditional Cardiac Devices Have Been at Risk of Malfunctioning or Overheating with MRIs. Heart Advisor, 14, 1, 11.

9. Nazarian, S., Hansford, R., Roguin, A., Goldsher, D., Zviman, M.M., Lardo, A.C., et al. (2011) A Prospective Evaluation of a Protocol for Magnetic Resonance Imaging of Patients with Implanted Cardiac Devices. Annals of Internal Medicine, 155, 415-424. https://doi.org/10.7326/0003-4819-155-7-201110040-00004

10. Russo, R.J., Costa, H.S., Silva, P.D., Anderson, J.L., Arshad, A., Biederman, R.W., et al. (2017) Assessing the Risks Associated with MRI in Patients with a Pacemaker or Defibrillator. The New England Journal of Medicine, 376, 755-764. https://doi.org/10.1056/NEJMoa1603265

11. Russo, R.J. (2013) Determining the Risks of Clinically Indicated Nonthoracic Magnetic Resonance Imaging at $1.5 \mathrm{~T}$ for Patients with Pacemakers and Implantable Cardioverter-Defibrillators: Rationale and Design of the MagnaSafe Registry. American Heart Journal, 165, 266-272. https://doi.org/10.1016/j.ahj.2012.12.004

12. Nazarian, S., Hansford, R., Rahsepar, A.A., Weltin, V., McVeigh, D., Gucuk Ipek, E., et al. (2017) Safety of Magnetic Resonance Imaging in Patients with Cardiac Devices. The New England Journal of Medicine, 377, 2555-2564. https://doi.org/10.1056/NEJMoa1604267

13. Samar, H., Yamrozik, J.A., Williams, R.B., Doyle, M., Shah, M., Bonnet, C.A., et al. (2017) Diagnostic Value of MRI in Patients with Implanted Pacemakers and Implantable Cardioverter-Defibrillators across a Cross Population. Does the Benefit Justify the Risk? A Proof of Concept Study. JACC: Clinical Electrophysiology, 3, 991-1002. https://doi.org/10.1016/j.jacep.2017.03.009

14. Guinand, A., Noble, S., Frei, A., Renard, J., Tramer, M.R. and Burri, H. (2016) Extra-Cardiac Stimulators: What Do Cardiologists Need to Know? Europace, 18, 1299-1307. https://doi.org/10.1093/europace/euv453 
15. Indik, J.H., Gimbel, J.R., Abe, H., Alkmim-Teixeira, R., Birgersdotter-Green, U., Clarke, G.D., et al. (2017) HRS Expert Consensus Statement on Magnetic Resonance Imaging and Radiation Exposure in Patients with Cardiovascular Implantable Electronic Devices. Heart Rhythm, 14, e97-e153. https://doi.org/10.1016/j.hrthm.2017.04.025

16. Centers for Medicare \& Medicaid Services (2018) Proposed Decision Memo for Magnetic Resonance Imaging (MRI) (CAG-00399R4).

17. Roguin, A., Zviman, M.M., Meininger, G.R., Rodrigues, E.R., Dickfeld, T.M., Bluemke, D.A., Lardo, A., Berger, R.D., Calkins, H. and Halperin, H.R. (2004) Modern Pacemaker and Implantable Cardioverter/Defibrillator Systems Can Be Magnetic Resonance Imaging Safe: In Vitro and in Vivo Assessment of Safety and Function at 1.5 T. Circulation, 110, 475-482. https://doi.org/10.1161/01.CIR.0000137121.28722.33

18. Poh, P.G., Liew, C., Yeo, C., Chong, L.R., Tan, A. and Poh, A. (2017) Cardiovascular Implantable Electronic Devices: A Review of the Dangers and Difficulties in MR Scanning and Attempts to Improve Safety. Insights Imaging, 8, 405-418. https://doi.org/10.1007/s13244-017-0556-3

19. Gimbel, J.R. and Wilkoff, B.L. (2003) Artefact Mimicking Tachycardia during Magnetic Resonance Imaging in a Patient with an Implantable Loop Recorder. Heart, 89, e10. https://doi.org/10.1136/heart.89.3.e10

20. Gimbel, J.R., Zarghami, J., Machado, C. and Wilkoff, B.L. (2005) Safe Scanning, But Frequent Artifacts Mimicking Bradycardia and Tachycardia during Magnetic Resonance Imaging (MRI) in Patients with an Implantable Loop Recorder (ILR). Annals of Noninvasive Electrocardiology, 10, 404-408.

https://doi.org/10.1111/j.1542-474X.2005.00056.x

21. Hong, S.N., Rahimi, A., Kissinger, K.V., Pedrosa, I., Manning, W.J. and O'Halloran, T.D. (2010) Cardiac Magnetic Resonance Imaging and the WATCHMAN Device. Journal of the American College of Cardiology, 55, 2785. https://doi.org/10.1016/j.jacc.2010.03.027

22. Langman, D.A., Goldberg, I.B., Finn, J.P. and Ennis, D.B. (2011) Pacemaker Lead Tip Heating in Abandoned and Pacemaker-Attached Leads at 1.5 Tesla MRI. Journal of Magnetic Resonance Imaging, 33, 426-431.

https://doi.org/10.1002/jmri.22463

23. Kim, J., Hwang, J., Choi, J.H., Choi, H.I., Kim, M.S., Jung, S.H., et al. (2017) Frequency and Clinical Impact of Retained Implantable Cardioverter Defibrillator Lead Materials in Heart Transplant Recipients. PLoS ONE, 12, e0176925. https://doi.org/10.1371/journal.pone.0176925

24. Martin, A., Voss, J., Shannon, D., Ruygrok, P. and Lever, N. (2014) Frequency and Sequelae of Retained Implanted Cardiac Device Material Post Heart Transplantation. Pacing and Clinical Electrophysiology, 37, 242-248. https://doi.org/10.1111/pace.12274

25. Mattei, E., Gentili, G., Censi, F., Triventi, M. and Calcagnini, G. (2015) Impact of Capped and Uncapped Abandoned Leads on the Heating of an MR-Conditional Pacemaker Implant. Magnetic Resonance in Medicine, 73, 390-400. https://doi.org/10.1002/mrm.25106

26. Henderson, J.M., Tkach, J., Phillips, M., Baker, K., Shellock, F.G. and Rezai, A.R. (2005) Permanent Neurological Deficit Related to Magnetic Resonance Imaging in a Patient with Implanted Deep Brain Stimulation Electrodes for Parkinson's Disease: Case Report. Neurosurgery, 57, E1063. https://doi.org/10.1227/01.NEU.0000180810.16964.3E

27. Blaschke, F., Lacour, P., Walter, T., Wutzler, A., Huemer, M., Parwani, A., et al. (2016) Cardiovascular Magnetic Resonance Imaging in Patients with an Implantable Loop Recorder. Annals of Noninvasive Electrocardiology, 21, 319-324. https://doi.org/10.1111/anec.12333

28. Higgins, J.V., Gard, J.J., Sheldon, S.H., Espinosa, R.E., Wood, C.P., Felmlee, J.P., et al. (2014) Safety and Outcomes of Magnetic Resonance Imaging in Patients with Abandoned Pacemaker and Defibrillator Leads. Pacing and Clinical Electrophysiology, 37, 1284-1290. https://doi.org/10.1111/pace.12419 
29. Strach, K., Naehle, C.P., Muhlsteffen, A., Hinz, M., Bernstein, A., Thomas, D., et al. (2010) Low-Field Magnetic Resonance Imaging: Increased Safety for Pacemaker Patients? Europace, 12, 952-960. https://doi.org/10.1093/europace/euq081

30. Hartnell, G.G., Spence, L., Hughes, L.A., Cohen, M.C., Saouaf, R. and Buff, B. (1997) Safety of MR Imaging in Patients Who Have Retained Metallic Materials after Cardiac Surgery. American Journal of Roentgenology, 168, 1157-1159. https://doi.org/10.2214/ajr.168.5.9129404

31. Murphy, K.J., Cohan, R.H. and Ellis, J.H. (1999) MR Imaging in Patients with Epicardial Pacemaker Wires. American Journal of Roentgenology, 172, 727-728. https://doi.org/10.2214/ajr.172.3.10063869

32. Kanal, E. (1998) Safety of MR Imaging in Patients with Retained Epicardial Pacer Wires. American Journal of Roentgenology, 170, 213-214. https://doi.org/10.2214/ajr.170.1.9423633

33. de Jonge, J.C., Melis, G.I., Gebbink, T.A., de Kort, G.A. and Leijten, F.S. (2014) Safety of a Dedicated Brain MRI Protocol in Patients with a Vagus Nerve Stimulator. Epilepsia, 55, e112-e115. https://doi.org/10.1111/epi.12774

34. Bohning, D.E., Lomarev, M.P., Denslow, S., Nahas, Z., Shastri, A. and George, M.S. (2001) Feasibility of Vagus Nerve Stimulation-Synchronized Blood Oxygenation Level-Dependent Functional MRI. Investigative Radiolo$g y, 36,470-479$. https://doi.org/10.1097/00004424-200108000-00006

35. Laible, M., Mohlenbruch, M., Horstmann, S., Pfaff, J., Geis, N.A., Pleger, S., et al. (2017) Peri-Procedural Silent Cerebral Infarcts after Left Atrial Appendage Occlusion. European Journal of Neurology, 24, 53-57. https://doi.org/10.1111/ene.13129

36. Mohrs, O.K., Wunderlich, N., Petersen, S.E., Pottmeyer, A. and Kauczor, H.U. (2011) Contrast-Enhanced CMR in Patients after Percutaneous Closure of the Left Atrial Appendage: A Pilot Study. Journal of Cardiovascular Magnetic Resonance, 13, 33. https://doi.org/10.1186/1532-429X-13-33

37. Padmanabhan, D., Kella, D.K., Mehta, R., Kapa, S., Deshmukh, A., Mulpuru, S., Jaffe, A.S., Felmlee, J.P., Jondal, M.L., Dalzell, C.M., Asirvatham, S.J., Cha, Y.M., Watson, R.E. and Friedman, P.A. (2018) Safety of Magnetic Resonance Imaging in Patients with Legacy Pacemakers and Defibrillators and Abandoned Leads. Heart Rhythm, 15, 228-233. https://doi.org/10.1016/j.hrthm.2017.10.022

38. Chouliaras, G., Berdoukas, V., Ladis, V., Kattamis, A., Chatziliami, A., Fragodimitri, C., et al. (2011) Impact of Magnetic Resonance Imaging on Cardiac Mortality in Thalassemia Major. Journal of Magnetic Resonance Imaging, 34, 56-59. https://doi.org/10.1002/jmri.22621

39. Centers for Medicare \& Medicaid Services (2011) Decision Memo for Magnetic Resonance Imaging (MRI) (CAG-00399R2).

40. Kramer, D.B. and Kesselheim, A.S. (2017) Coverage of Magnetic Resonance Imaging for Patients with Cardiac Devices: Improving the Coverage with Evidence Development Program. JAMA Cardiology, 2, 711-712. https://doi.org/10.1001/jamacardio.2017.1674

41. Acha, M.R., Keaney, J.J., Lubitz, S.A., Milan, D.J., Mansour, M., Heist, K.E., et al. (2015) Increased Perforation Risk with an MRI-Conditional Pacing Lead: A Single-Center Study. Pacing and Clinical Electrophysiology, 38, 334-342. https://doi.org/10.1111/pace.12550

42. Elmouchi, D.A., Rosema, S., Vanoosterhout, S.M., Khan, M., Davis, A.T., Gauri, A.J., et al. (2014) Cardiac Perforation and Lead Dislodgement after Implantation of a MR-Conditional Pacing Lead: A Single-Center Experience. Pacing and Clinical Electrophysiology, 37, 4-10. https://doi.org/10.1111/pace.12293 


\section{ABBREVIATIONS}

MRI Magnetic Resonance Imaging

ACR American College of Radiology

CIED Cardiac Implantable Electronic Device

PM Pacemaker

ICD Implantable Cardioverter Defibrillator

LAAO Left Atrial Appendage Occlusion device

SAR Specific Absorbance Rate

ILR Implantable Loop Recorder

VNS Vagal Nerve Stimulator

BOLD fMRI Blood Oxygenation Level-Dependent functional MR Imaging

CMS Centers for Medicare and Medicaid Services

NCD National Coverage Determination 